Few-Body Systems Suppl. 0, 1-4 (2018)

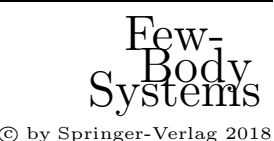

(c) by Springer-Verlag 2018 Printed in Austria

\title{
Axial Excitation of the $\Delta$ in Chiral Quark Models
}

\author{
S. Šrca ${ }^{1,2}$, L. Amoreira ${ }^{3,4}$, M. Fiolhais ${ }^{5,4}$, B. Golli ${ }^{6,2}$ \\ ${ }^{1}$ Faculty of Mathematics and Physics, University of Ljubljana, Slovenia \\ 2 Jožef Stefan Institute, Ljubljana, Slovenia \\ ${ }^{3}$ Department of Physics, University of Beira Interior, Covilhã, Portugal \\ ${ }^{4}$ Centre for Computational Physics, University of Coimbra, Portugal \\ ${ }^{5}$ Department of Physics, University of Coimbra, Portugal \\ ${ }^{6}$ Faculty of Education, University of Ljubljana, Slovenia
}

\begin{abstract}
We discuss electro-magnetic and weak axial N- $\Delta$ transition amplitudes in the linear $\sigma$-model and the Cloudy-Bag Model as typical representatives of chiral quark models. We show that good qualitative understanding of the transition can be obtained in models which, in addition to the pion cloud, incorporate a fluctuating $\sigma$-field inside the baryon.
\end{abstract}

\section{Introduction}

The present work was partly motivated by the experience gained in the phenomenological description of the quadrupole electro-excitation of the $\Delta$ within the linear $\sigma$-model (LSM) and the Chromo-Dielectric Model (CDM) in which the pionic degree of freedom was shown to play a dominant role [1]. In these models, the pion cloud practically saturates the electric and Coulomb quadrupole transition strengths and qualitatively reproduces the $Q^{2}$-behaviour of the amplitudes. Furthermore, the results for the ratios of electro-magnetic couplings E2/M1 and C2/M1 at $Q^{2}=0$ were found to be rather insensitive to the details of the models. In the LSM, the absolute values of the transverse helicity amplitudes were well reproduced, although the underprediction of the strength at $Q^{2}=0$ remains an open question. To pin down the pertinent model ingredients, one therefore needs to probe non-zero values of $Q^{2}$ where effects of a possibly strong pion cloud in the interiors of the nucleon and the $\Delta$ are manifested differently.

We anticipate that the theoretical investigation of axial $\mathrm{N}-\Delta$ transition amplitudes [2] in chiral quark models may reveal additional information on non-quark degrees of freedom in baryons. Yet due to difficulties in consistent 
incorporation of the pion field, the model predictions for these amplitudes are very scarce [3], in contrast to the electro-magnetic sector. Experimentally, the structure of the weak axial transition currents is explored by using weak probes [4] or electron scattering [5]. We have a stable world-average for the dominant coupling $C_{5}^{\mathrm{A}}(0)[6]$, but a very poor knowledge of $C_{3}^{\mathrm{A}}(0), C_{4}^{\mathrm{A}}(0)$, and the corresponding form-factors. We calculated the axial amplitudes in the Cloudy-Bag Model (CBM) and in the LSM.

\section{Calculation of the amplitudes}

In the linear $\sigma$-model and related classes of models involving quarks interacting with chiral fields $\sigma$ and $\vec{\pi}$ the Hamiltonian can be written as

$$
H=H_{q}^{0}+H_{\sigma}+\int \mathrm{d} \boldsymbol{r}\left\{\frac{1}{2}\left[\vec{P}_{\pi}^{2}+\left(\nabla^{2}+m_{\pi}^{2}\right) \vec{\pi}^{2}\right]+U(\sigma, \vec{\pi})+\vec{j} \vec{\pi}\right\},
$$

where $j_{a}$ is the quark source, $\vec{P}_{\pi}$ is the pion conjugate momentum, $H_{q}^{0}$ and $H_{\sigma}$ are the free-quark and the $\sigma$-meson terms, and $U(\sigma, \vec{\pi})$ is the meson selfinteraction term. In the Cloudy-Bag Model the $\sigma$-field and the $U$-term are absent, while in the linear $\sigma$-model all terms are present and $U$ is the wellknown Mexican-hat potential. The CDM has an additional scalar-isoscalar field which mimics the glueballs of QCD and dynamically confines the quarks [7].

Evaluating the commutator $\left[H, \vec{P}_{\pi}\right]$ between eigenstates of the Hamiltonian $|\mathrm{N}\rangle$ and $|\Delta\rangle$ (regardless of the model) we obtain a virial constraint of the form

$$
\left(-\Delta+m_{\pi}^{2}-\left(E_{\Delta}-E_{\mathrm{N}}\right)^{2}\right)\left\langle\Delta\left|\pi_{0}(\boldsymbol{r})\right| \mathrm{N}\right\rangle=-\left\langle\Delta\left|J_{0}(\boldsymbol{r})\right| \mathrm{N}\right\rangle
$$

where the source on the RHS of consists of the quark term and the term originating from the meson self-interaction (if present):

$$
J_{0}(\boldsymbol{r})=j_{0}(\boldsymbol{r})+\frac{\partial U(\sigma, \vec{\pi})}{\partial \pi_{0}(\boldsymbol{r})} .
$$

In the CBM we assume the usual perturbative form for the pion profiles using the experimental masses for the nucleon and $\Delta$, which fulfills (1). The method we used to impose this constraint in the LSM is described in refs. [8, 9].

In computing the transverse $\left(\tilde{A}^{\mathrm{A}}\right)$, longitudinal $\left(\tilde{L}^{\mathrm{A}}\right)$, and scalar $\left(\tilde{S}^{\mathrm{A}}\right)$ transition helicity amplitudes between states with definite four-momenta, we interpret our localised model states as wave packets of states with good linear momentum. Extending the method explained in [10] we find, to order $k^{2} / M^{2}$ :

$$
\begin{aligned}
C_{6}^{\mathrm{A}} & =\frac{M_{\mathrm{N}}^{2}}{k^{2}}\left[-\tilde{A}_{\frac{3}{2}}^{\mathrm{A}}+\sqrt{\frac{3}{2}} \tilde{L}^{\mathrm{A}}\right] \frac{2 M_{\Delta}}{M_{\Delta}+M_{\mathrm{N}}}, \\
C_{5}^{\mathrm{A}} & =-\sqrt{\frac{3}{2}}\left(\tilde{L}^{\mathrm{A}}-\frac{k_{0}}{k} \tilde{S}^{\mathrm{A}}\right) \frac{2 M_{\Delta}}{M_{\Delta}+M_{\mathrm{N}}}-\frac{k_{0}^{2}-k^{2}}{M_{\mathrm{N}}^{2}} C_{6}^{\mathrm{A}}, \\
C_{4}^{\mathrm{A}} & =\frac{M_{\mathrm{N}}^{2}}{k M_{\Delta}}\left[-\sqrt{\frac{3}{2}} \tilde{S}^{\mathrm{A}}+\frac{k_{0} k}{M_{\mathrm{N}}^{2}} \frac{M_{\Delta}+M_{\mathrm{N}}}{2 M_{\Delta}} C_{6}^{\mathrm{A}}\right]-\frac{M_{\mathrm{N}}^{2}}{2 M_{\Delta}^{2}} C_{5}^{A} .
\end{aligned}
$$


For a finite pion mass, the divergence of the axial transition current is given by the PCAC relation $\left\langle\Delta^{+}\left(p^{\prime}\right)\left|\partial^{\alpha} A_{\alpha a}\right| \mathrm{N}^{+}(p)\right\rangle=-m_{\pi}^{2} f_{\pi}\left\langle\Delta^{+}\left(p^{\prime}\right)\left|\pi_{a}(0)\right| \mathrm{N}^{+}(p)\right\rangle$, where $a$ is the isospin index and the transition matrix element of the pion field with $a=0$ is related to the strong form factor by

$$
\left\langle\Delta^{+}\left(p^{\prime}\right)\left|\pi_{0}(0)\right| \mathrm{N}^{+}(p)\right\rangle=\mathrm{i} \frac{G_{\pi \Delta N}\left(Q^{2}\right)}{2 M_{\mathrm{N}}} \frac{\bar{u}_{\Delta \mu} q^{\mu} u_{\mathrm{N}}}{Q^{2}+m_{\pi}^{2}} \sqrt{\frac{2}{3}} .
$$

Assuming that the pion pole dominates the $C_{6}^{\mathrm{A}}\left(Q^{2}\right)$ amplitude for $Q^{2} \rightarrow-m_{\pi}^{2}$, the resulting off-diagonal Goldberger-Treiman relation $[2,10,11]$ offers an alternative method to compute $C_{5}^{\mathrm{A}}\left(Q^{2}\right)$ from the strong $G_{\pi \mathrm{N}} \Delta$ form-factor,

$$
C_{5}^{\mathrm{A}}\left(Q^{2}\right)=f_{\pi} \frac{G_{\pi \mathrm{N} \Delta}\left(Q^{2}\right)}{2 M_{\mathrm{N}}} \sqrt{\frac{2}{3}}=\frac{2 M_{\Delta}}{M_{\Delta}+M_{N}} \sqrt{\frac{2}{3}} \frac{f_{\pi}}{\mathrm{i} k} \int \mathrm{d} \boldsymbol{r} e^{\mathrm{i} \boldsymbol{k} \boldsymbol{r}}\left\langle\Delta\left\|J_{0}(\boldsymbol{r})\right\| \mathrm{N}\right\rangle .
$$

\section{Results and discussion}

The calculated $C_{5}^{\mathrm{A}}(0)$ is $25 \%$ higher than the experimental average, but the $Q^{2}$-dependence is reproduced to within a few percent in terms of the dipole cut-off parameter. A better result at $Q^{2}=0$ can be obtained by determining $C_{5}^{\mathrm{A}}\left(Q^{2}\right)$ from the calculated strong $\pi \mathrm{N} \Delta$ form-factor through the off-diagonal Goldberger-Treiman relation, yet the $Q^{2}$-dependence becomes steeper, with a cut-off of $\approx 0.80 \mathrm{GeV}$. The disagreement between the two approaches can be attributed to an overestimate of the meson strength, a characteristic feature of LSM where only the meson fields bind the quarks. (Still, the effect of the meson self-interaction is relatively weak in the strong coupling constants.) Essentially the same trend is observed in the nucleon case where we obtain $g_{\mathrm{A}}=1.41$. The discrepancy with respect to the experimental value of 1.27 is commensurate with the disagreement in $C_{5}^{\mathrm{A}}(0)$. Unfortunately, the overestimate of $g_{\mathrm{A}}$ and $G_{\mathrm{A}}\left(Q^{2}\right)$ in the LSM seems to persist even if the spurious centre-of-mass motion of the nucleon is removed [12].

In the CBM the picture is reversed. As it has been shown in ref. [8], only the quarks contribute to the $C_{4}^{\mathrm{A}}$ and $C_{5}^{\mathrm{A}}$ amplitudes, while $C_{6}^{\mathrm{A}}$ is almost completely dominated by the pion pole. Hence the calculated values of $C_{5}^{\mathrm{A}}(0)$ are too small, ranging from $68 \%$ of the experimental estimate at $R=0.7 \mathrm{fm}$ to only $56 \%$ at $R=1.3 \mathrm{fm}$. The behaviour of $C_{5}^{\mathrm{A}}\left(Q^{2}\right)$ is similar as in the pure MIT Bag Model (to within $10 \%$ ), with a fitted dipole cut-off of $\sim 1.2 \mathrm{GeV} \mathrm{fm} / R$. The off-diagonal Goldberger-Treiman relation is satisfied in the $\mathrm{CBM}$, but $C_{5}^{\mathrm{A}}\left(Q^{2}\right)$ calculated from $G_{\pi \mathrm{N} \Delta}\left(Q^{2}\right)$ has a steeper $Q^{2}$-dependence with a cut-off of $\sim$ $0.8 \mathrm{GeV} \mathrm{fm} / R$. The large discrepancy can be partly attributed to the fact that the CBM predicts a too low value for $G_{\pi \mathrm{NN}}$, and consequently for $G_{\pi \mathrm{N} \Delta}$.

The determination of the $C_{4}^{\mathrm{A}}$ is less reliable because the meson contribution to the scalar amplitude is very sensitive to small variations of the profiles. However, the experimental value is very uncertain as well. Neglecting the nonpole contribution to $\tilde{S}^{\mathrm{A}}$ and $C_{6}^{\mathrm{A}}$, the value of $C_{4}^{\mathrm{A}}$ is dominated by the term $-\left(M_{\mathrm{N}}^{2} / 2 M_{\Delta}^{2}\right) C_{5}^{\mathrm{A}}$, in agreement with the popular value of $C_{4}^{\mathrm{A}}(0)=-0.3$. 
In accordance with our experience in the electro-magnetic sector, we find that the quark contribution alone strongly underestimates the $C_{5}^{\mathrm{A}}$ amplitude. If only a linear coupling of pions to quarks is added, the situation does not improve since in such a case the pion contribution to $C_{5}^{\mathrm{A}}$ vanishes. On the other hand, the inclusion of meson self-interaction which allows for a substantial deviation of the $\sigma$-field from its vacuum value inside the baryon considerably increases $C_{5}^{\mathrm{A}}$. The LSM overestimates this contribution, but this is not the case in other chiral models which allow for a non-zero fluctuation of the $\sigma$-field; in a version of the NJL model with nonlocal regulators [13] the contribution of sea quarks to the nucleon $g_{\mathrm{A}}$ is more than a factor of two weaker than the equivalent contribution of chiral mesons in the LSM.

This work was supported by FCT (POCTI/FEDER), Lisbon, and by The Ministry of Science and Education of Slovenia.

\section{References}

1. M. Fiolhais, B. Golli, S. Širca: Phys. Lett. B373, 229 (1996)

2. C.H. Llewellyn Smith: Phys. Rep. 3C, 261 (1972)

3. Jùn Líu, N.C. Mukhopadhyay, L. Zhang: Phys. Rev. C52, 1630 (1995)

4. S.J. Barish et al.: Phys. Rev. D19, 2521 (1979); G.M. Radecky et al.: Phys. Rev. D25, 1161 (1982); T. Kitagaki et al.: Phys. Rev. D42, 1331 (1990), and references therein

5. A. Liesenfeld et al.: Phys. Lett. B468, 20 (1999), and references therein; L. Elouadrhiri, D. Heddle (spokespersons): JLab Proposal E94-005; N. Simićević, S. P. Wells (spokespersons): JLab Proposal PR97-104

6. N.C. Mukhopadhyay et al.: Nucl. Phys. A633, 481 (1998)

7. T. Neuber, M. Fiolhais, K. Goeke, J.N. Urbano: Nucl. Phys. A560, 909 (1993)

8. B. Golli, S. Širca, L. Amoreira, M. Fiolhais: submitted to Phys. Lett. B

9. B. Golli, L. Amoreira, M. Fiolhais, S. Širca: Bled Workshops in Physics 3, 47 (2002); hep-ph/0211293; S. Širca, L. Amoreira, M. Fiolhais, B. Golli: Bled Workshops in Physics 3, 67 (2002); hep-ph/0211292

10. T.R. Hemmert, B.R. Holstein, N.C. Mukhopadhyay: Phys. Rev. D51, 158 (1995)

11. P.A. Schreiner, F. von Hippel: Nucl. Phys. B58, 333 (1973)

12. M. Rosina, M. Fiolhais, B. Golli, S. Širca: In: Proceedings of the Meeting "Nuclear and Particle Physics with CEBAF at Jefferson Lab", November 3-10, 1998, Dubrovnik, Croatia, Fizika B8, 383 (1999)

13. W. Broniowski, B. Golli, G. Ripka: Nucl. Phys. A703, 667 (2002) 